\title{
Interactive comment on "A mechanistic classification of double tides" by J. A. Mattias Green et al.
}

\section{Anonymous Referee \#1}

Received and published: 28 July 2018

\section{General comments}

This is a well-structured manuscript, well-illustrated and referenced, though for a reviewer there are several irritating editorial blunders.

However, the authors overate the scientific interest in tidal double high and low waters, which are part of a wider context of higher harmonics distorting pure tidal cosine curves. A volume celebrating 100 Years of Liverpool Tidal Institute should hope for more significant contributions. This paper needs much more physical interpretation and scientific insight: we need more about the dynamics of Kelvin waves and Standing waves.

Classically Double Tides are found where an ingoing Kelvin wave and a weakened 
outgoing reflected Kelvin wave interfere. Any classification must evolve from this basic well-understood hydrodynamics. Almost always the tidal ranges are small. Oddly, one of the best known and understood of these DT locations, Courtown on the eastern Irish coast, is not even mentioned in the paper, though very near to Bangor University!

In summary, If space is limited then it has a lower priority. But it could be published after substantial additional work if Editors have space available

Interactive comment

Specific Comments

The paper has "mechanistic" in the title, which encapsulates the authors' approach. This is an exercise in number generation from a global tidal model, rather than development of a physical insight.

The elevation of a small piece of algebra in Doodson's Manual, to a "Doodson Criteria" is excessive. Doodson, surely the greatest contributor to the LTI -100 reputation, only showed an absolute minimum condition for DTs. Somehow the authors have set the "Criterion" up, so they can convieniently elaborate it by introducing phase relationships as a further refinement. . .more a complication than a new perspective.

Any discussion of DTs should look also at the dynamics of currents in the vicinity.

As the generation of shallow-water harmonics is a highly non-linear process, so too is their effect in distorting tidal curves. This is well covered in existing textbooks, despite the authors' assertion otherwise. And as a consequence of the non-linearity, DTs are much more evident at spring tides than at neaps. The paper should acknowledge this and deal with the temporal variations in DT occurrences (they do briefly and superficially for the 18.6 year nodal variations at the end of the paper) The other spring-neap influence on multiple HW/LW is the movement of amphidromes, as shown at Courtown (see above). The spring-neap effects on shallow water tides is basically caught by the MS4 harmonic constituent, which is not included in this paper.

Abstract L8 harmonics FIT to tidal curves. They do not "explain" in any physical sense. 
L18 surely given the resolution of the model, these 8000 potential locations are generally in a much smaller set of local clusters.

OSD

L19 a curious use of "manual work"

L23 not per se especially scientifically interesting

L31 "transient coherent seiche"

\section{L34 "Provides"}

L37 and of course Courtown

\section{L43 b/a}

L53 discuss the dynamics behind this node/antinode configuration

This three classification categories are not robustly independent, so many cases may be a mixture of two or all three trigonometric criteria.

L63 "for the harmonic amplitude conditions"

L68 misuse of "still" . delete.

A more direct approach would be to examine a global tidal map for standing waves reflected in shallow water. (Remembering of course the spring-neap cycle)

L76 These are very small M2 tides, surely often hidden in non-tidal sea level changes. Classes 1 and 2 have a linked dynamic interpretation in terms of Kelvin waves, of which the Authors seem unaware.

L78 This thirs criterion misses the case where a tidal standing wave, say M4, has a node at the entrance, so zero M4 amplitude but strong M4 currents.

L85 MS4 (and maybe eg combinations of $(M+S) 6$ etc) is essential here.

L01 Were these all independent dynamically? Could list.
Interactive

comment
Printer-friendly version

Discussion paper 
L13 page 3 needs discussing in Kelvin Wave dynamics, relating to "the nature of the tide in the North Sea". It's the weaker reflected wave phenomena again.

OSD

Page 4 L53 does acknowledge the transient nature of DTs, but should consider these in relation to the S/N cycle. MS4 must be included.

L63 could be an amplitude node for M6. . but not for currents. M4 , M6 resonances could lead to such patterns.

\section{L68 suffixes missing}

L70 Providence . "likely to be the " but this is a weak unsupported assertion.

L80 S/N much more important influence than Nodal effects.

L84 These are non-linear processes so the scaling is not linear. This argument needs refining or removing. Remember that the theoretical $3.7 \%$ nodal modulation is reduced to ca $2.2 \%$ in many places due to non-linear energy dissipation.

\section{L89 Table 1}

L 94 could refer to $N^{\star *} 2$ here and comment that $n=2$ still applies!

Page 6 L01 Omit this hand waving argument or clarify.."this may exaggerate" is very weak.

L11 A sweeping and erroneous generalisation, Although for illustration the M2/M4 example has merit in text books. Students do not need further complications, only maybe needing to be aware of the local nature of higher-harmonic generation. DTs occur in time not frequency space.

L17 delete "at the end of the day" jargon.

L22 on the climate change band wagon. Even the main tides are little affected by realistic sea level increases.

The Figures and Tables are ok, except that curves are clipped top and bottom in Figure
Interactive

comment
Printer-friendly version

Discussion paper 
3.

Interactive comment on Ocean Sci. Discuss., https://doi.org/10.5194/os-2018-72, 2018.
OSD

Interactive comment 